\title{
Physics of zonal flows
}

\begin{tabular}{|l|l|}
\hline $\begin{array}{l}\text { jour nal or } \\
\text { publ i cat i on } \mathrm{title}\end{array}$ & Physi cs of Pl asmas \\
\hline vol une & Vol .13 \\
\hline number & I ssue 5 \\
\hline page range & pp. 055502-1 - 055502-11 \\
\hline year & 2006- 05- 01 \\
\hline URL & ht t p: //hdl . handl e. net /10655/2254 \\
\hline
\end{tabular}


PHYSICS OF PLASMAS 13, 055502 (2006)

\title{
Physics of zonal flows ${ }^{a)}$
}

\author{
K. Itoh ${ }^{\text {b) }}$ \\ National Institute for Fusion Science, Toki, 509-5292, Japan \\ S.-I. Itoh \\ Research Institute for Applied Mechanics, Kyushu University, Kasuga 816-8580, Japan \\ P. H. Diamond \\ University of California at San Diego, La Jolla, California 92093-0319
}

T. S. Hahm

Princeton Plasma Physics Laboratory, P.O. Box 451, Princeton, New Jersey 08543-0451

A. Fujisawa

National Institute for Fusion Science, Toki, 509-5292, Japan

G. R. Tynan

University of California at San Diego, La Jolla, California 92093-0319

M. Yagi and Y. Nagashima

Research Institute for Applied Mechanics, Kyushu University, Kasuga 816-8580, Japan

(Received 28 October 2005; accepted 10 January 2006; published online 15 May 2006)

\begin{abstract}
Zonal flows, which means azimuthally symmetric band-like shear flows, are ubiquitous phenomena in nature and the laboratory. It is now widely recognized that zonal flows are a key constituent in virtually all cases and regimes of drift wave turbulence, indeed, so much so that this classic problem is now frequently referred to as "drift wave-zonal flow turbulence." In this review, new viewpoints and unifying concepts are presented, which facilitate understanding of zonal flow physics, via theory, computation and their confrontation with the results of laboratory experiment. Special emphasis is placed on identifying avenues for further progress. (C) 2006 American Institute of Physics. [DOI: 10.1063/1.2178779]
\end{abstract}

\section{INTRODUCTION}

This article presents a critical assessment of the physics of zonal flows and their relevance to fusion plasmas. This assessment is based on a comprehensive review of zonal flow physics, ${ }^{1,2}$ recently published and on an overview talk. ${ }^{3}$ In this article, we focus on:

(a) presenting the current state of our understanding of zonal flow physics, i.e., describing and delineating what we understand, what we think we understand but need more research on, and what we do not understand,

(b) discussing the importance of zonal flow physics for magnetic fusion, and

(c) describing rapid development in experiments which have examined zonal flow physics.

The constitution of this article is as follows. First, the basic physics of zonal flows is explained in Sec. II. The importance of zonal flows for magnetic confinement fusion research is discussed in Sec. III. An assessment of our present understanding is given in Sec. IV. Experimental progress is surveyed in Sec. V. Future research is suggested in Sec. VI, and a summary is made in Sec. VII. Due to the length limit, this article is necessarily quite terse. For a more

\footnotetext{
a) Paper JR1 1, Bull. Am. Phys. Soc. 50, 179 (2005).

${ }^{b)}$ Invited speaker.
}

detailed discussion, please refer to the full-length review, Refs. 1 and 2, and references therein.

\section{BASIC PHYSICS OF ZONAL FLOWS}

Zonal flows (ZFs) are $n=0, m \cong 0$ electric field fluctuations with finite radial wave number $q_{r}(m, n$ : poloidal and toroidal mode numbers, respectively). Thus, zonal flows cannot drive radial transport and so cannot tap the usual free energy sources $(\nabla n, \nabla T$, etc.). As a consequence, zonal flows must be "pumped" by nonlinear interactions. Zonal flows coexist with, and are excited by all types of microinstabilities [hereafter generically dubbed "drift waves" (DWs)] and regulate transport by shearing the underlying drift waves, thus quenching them by extracting energy from them. For this reason, zonal flows are a crucial player in the selfregulation mechanism for drift wave turbulence and transport, so much so that this type of turbulence is now commonly referred to as drift wave-zonal flow turbulence, as depicted in Fig. 1.

\section{A. Characteristic features of zonal flows}

As noted previously, much of the interest in zonal flows is driven by the fact that they regulate turbulence via shearing. However, it is certainly true that all low- $n$ modes in a spectrum of drift wave turbulence will shear and strain the higher- $n$, smaller-scale fluctuations. ${ }^{4,5}$ Indeed, nonlocal 


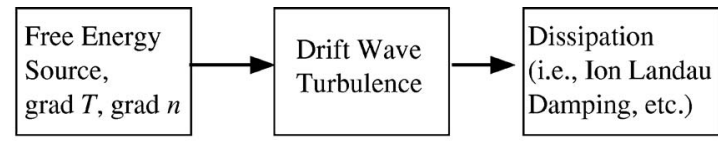

Classic Paradigm of Drift Wave Turbulence

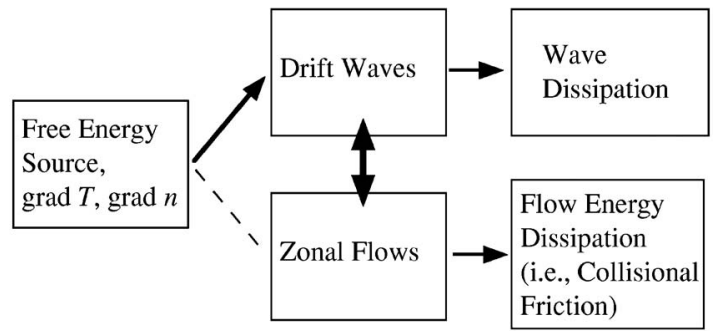

New Paradigm of Drift Wave-Zonal flow Turbulence

FIG. 1. New paradigm of drift wave turbulence with zonal flows.

shearing-straining interactions are characteristic of twodimensional (2D) turbulence once large scale vortices are established. This, in turn, naturally motivates the questions: "What is so special about zonal flows (with $n=0$ )?" and "Why aren't other low- $n$ modes given equal consideration as regulators of drift wave turbulence?" There are at least three answers to this very relevant and interesting question. These are discussed in the following.

First, zonal flows may be said to be the "modes of minimal inertia." This is because zonal flows, with $n=0$ and $k_{\|}$ $=0$, are not screened by Boltzmann electrons, as are the usual drift modes. Hence, the potential vorticity of a zonal flow mode is simply $q_{r}^{2} \rho_{s}^{2} \widetilde{\phi}_{\mathrm{ZF}}$, as opposed to $\left(1+k_{\perp}^{2} \rho_{s}^{2}\right) \widetilde{\phi}_{k}$ for drift waves, so that zonal flows have lower effective inertia than standard drift waves do. ( $\widetilde{\phi}_{\mathrm{ZF}}$ and $\widetilde{\phi}_{k}$ stand for potential fluctuations of ZFs and DWs, respectively.) The comparatively low effective inertia of zonal flows means that large zonal flow velocities develop in response to drift wave drive, unless damping intervenes. In this regard, it is also worthwhile to point out that in the case of electron temperature gradient (ETG) mode turbulence, both zonal flows and ETG modes excite a Boltzmann ion response $\tilde{n}_{i} / n_{0}=-|e| \widetilde{\phi} / T$, since $k_{\perp} \rho_{i} \gg 1$ for ETG. Hence, it is no surprise that zonal flow growth is slower for ETG turbulence than for its drift-ITG (ion temperature gradient) mode counterpart, since for ETG, zonal flows have an effective inertia comparable to that of other modes. ${ }^{6}$

Second, zonal flows, with $n=0$ and $k_{\|}=0$, are modes of minimal Landau damping. This means that the only linear dissipation acting on zonal flows for asymptotic times (i.e., $t \rightarrow \infty)$ is due to collisions. In particular, no linear, timeasymptotic dissipation acts on zonal flows in a collisionless system.

Third, since zonal flows have $n=0$, they are intrinsically incapable of driving radial $E \times B$ flow perturbations. Thus, they cannot tap expansion free energy stored in radial gradient. Thus, zonal flows do not cause transport or relaxation, and so constitute a benign repository for free energy. In contrast, other low $n$-modes necessarily involve a tradeoff be-

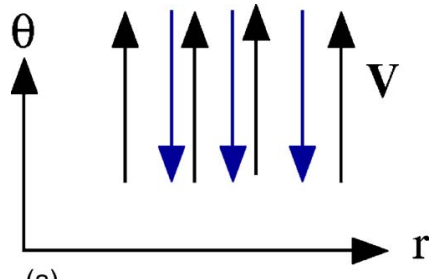

(a)

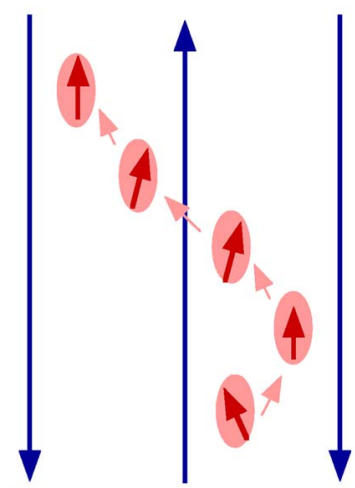

(b)

FIG. 2. (a) Zonal flows are illustrated and (b) motion of a drift wave packet in a trough of ZFs is schematically shown. Arrows on a packet denote the wave vector, indicating the modification of $k_{r}$.

tween shearing (a "plus" for confinement) and enhanced transport (a "minus").

\section{B. Excitation of zonal flows}

Having established the physics of shearing, it is illuminating to present a short "back-of-an-envelope" type demonstration of zonal flow instability. For other approaches, see the literature cited in Ref. 1. Consider a packet of drift waves propagating in an ensemble of quasistationary, random zonal flow shear layers, as shown in Fig. 2. Take the zonal flows as slowly varying with respect to the drift waves (i.e., $\Omega \ll \omega_{k}$ ), i.e., as quasistationary. Here, $\Omega$ is the rate of the change or frequency of the zonal flow and $\omega_{k}$ is the characteristic frequency of drift waves. [The motion of the drift wave packet is perturbed as is illustrated in Fig. 2(b).] The spatially complex shearing flow will result in an increase in $\left\langle k_{r}^{2}\right\rangle$, the mean square radial wave vector (i.e., consider a random walk of $k_{r}$, as described earlier). In turn the drift wave frequency $\omega_{e^{*}} /\left(1+k_{\perp}^{2} \rho_{s}^{2}\right)$ must then decrease. Here, $\rho_{s}$ is the ion gyroradius at the electron temperature. Since $\Omega \ll \omega_{k}$, the drift wave action density $N_{k}=E(k) / \omega_{k}$ is conserved, so that drift wave energy must also decrease. As the total energy of the system of waves and flows is also conserved (i.e., $E_{\text {wave }}$ $+E_{\text {flow }}=$ const), it thus follows that the zonal flow energy must, in turn, increase. Hence, the initial perturbation is reinforced, suggestive of instability. Note that the simplicity, clarity and universality of this argument support the assertion that zonal flow generation is a robust and ubiquitous phenomenon.

It is also important to clarify the distinction between zonal flows and the mean radial electric field. The latter is derived from a coarse-graining of the total electric field and varies smoothly in comparison to the zonal flow field. The 
zonal flow pattern is oscillatory and complex, exhibiting structure on scales of $10-20 \rho_{i}$. The mean $\left\langle E_{r}\right\rangle$ evolves on transport time scales, whereas the zonal flows can evolve on turbulence time scales (i.e., on a few correlation times). The mean electric field shear coherently stretches vortices, so that $\left\langle\delta k_{r}^{2}\right\rangle \sim k_{\theta}^{2}\left\langle V_{E}^{\prime}\right\rangle^{2} t^{2}$. By way of contrast, the zonal flows diffusively scatter $k_{r}$, so $\left\langle\delta k_{r}^{2}\right\rangle \sim D_{k} t$, where $D_{k} \sim k_{\theta}^{2}\left\langle\tilde{V}_{E}^{\prime 2}\right\rangle \tau_{c}$. Here $\left\langle\widetilde{V}_{E}^{\prime 2}\right\rangle$ is the mean square zonal shear and $\tau_{c}$ is the autocorrelation time of the zonal flow field. (The distinction between the inhomogeneous mean radial electric field and zonal flows is made here by the condition that the inhomogeneity scale length of mean electric field $L_{E}$ is much longer than $v_{\mathrm{gr}} / \Delta \omega$ and that the electric field changes more slowly than the rate $v_{\mathrm{gr}} / L_{E}$, where $v_{\mathrm{gr}}$ and $\Delta \omega$ are characteristic values of the radial group velocity and decorrelation rate of drift waves, respectively.) Finally, the mean electric field is driven by heating, fueling, momentum input, etc. (which determines the equilibrium profiles, which in turn regulate radial force balance), whereas zonal flows are driven exclusively by nonlinear wave interaction processes.

\section{Self-regulation of the DW-ZF system}

A key feature of zonal flows is their ability to coexist with drift waves, and so form a self-regulating system. The drift wave zonal flow system can be described by a generalized predator-prey model for the evolution of $W_{d}$, the drift wave energy, and $W_{\mathrm{ZF}}$, the zonal flow energy. Despite wide variability in the basic models and details of the system (which are extensively discussed in Sec. 3 of Ref. 1), the evolution equations have a universal structure of the form

$$
\begin{aligned}
& \frac{\partial W_{d}}{\partial t}=\gamma\left[\nabla P_{0}, \ldots\right] W_{d}-\alpha W_{d} W_{\mathrm{ZF}}-\beta W_{d}^{2}, \\
& \frac{\partial W_{\mathrm{ZF}}}{\partial t}=-\gamma_{\mathrm{damp}}\left[\nu, \varepsilon_{T}, q\right] W_{\mathrm{ZF}}+\alpha W_{d} W_{\mathrm{ZF}}-\gamma_{\mathrm{NL}}\left(W_{\mathrm{ZF}}\right) W_{\mathrm{ZF}} .
\end{aligned}
$$

Here, $W_{d}=k^{2} L_{n}^{2}\left|e \widetilde{\phi}_{d} / T_{e}\right|^{2}$ and $W_{\mathrm{ZF}}=q_{r}^{4} k^{-2} L_{n}^{2}\left|e \widetilde{\phi}_{\mathrm{ZF}} / T_{e}\right|^{2}$ are normalized intensity of DWs and ZFs, $\left(\widetilde{\phi}_{d}\right.$ and $\widetilde{\phi}_{\mathrm{ZF}}$ are electrostatic potential perturbations of DWs and ZFs, $k$ is the characteristic wave number of DWs, $q_{r}$ is the radial wave number of ZFs, $L_{n}$ is the density gradient scale length), $\gamma\left[\nabla P_{0}, \ldots\right]$ is the drift wave growth rate (which is a function of the global parameters such as $\left.\nabla P_{0}\right), \gamma_{\text {damp }}$ is the linear zonal flow damping rate (which depends on the ion collision frequency $\nu_{i}$, inverse aspect-ratio $\varepsilon_{T}$, safety factor $q$, etc.), $\gamma_{\mathrm{NL}}\left(W_{\mathrm{ZF}}\right)$ is the nonlinear damping rate of zonal flows, in contrast to $\gamma_{\text {damp }}, \alpha$ is the coupling coefficient between DW and $\mathrm{ZFs}$, and $\beta$ stands for the coefficient of the nonlinear damping rate of DW. Note that drift waves and zonal flows can exchange energy through the term $\alpha W_{d} W_{\mathrm{ZF}}$. In the case where $\gamma_{\mathrm{NL}} \rightarrow 0, W_{d} \sim \gamma_{\mathrm{damp}} / \alpha$ so that the drift wave amplitude is set by zonal flow damping. Note that this damping is, in turn, set by the ion-ion collisionality and elements of the confinement geometry (i.e., via $\varepsilon_{T}, q$, etc.), so that zonal flow damping is thus identified as a "control knob" for the level of drift wave turbulence. As a consequence, the drift wave ther- mal diffusivity is reduced from the standard gyro-Bohm prediction by a factor $\mathcal{R} \sim \gamma_{\mathrm{damp}} / \alpha$, so $\chi \sim \mathcal{R} \chi_{g B}{ }^{7}$ Additional transport reduction effects, such as those related to the transport cross-phase, may enter the $\mathcal{R}$-factor, as well. Note that the $\mathcal{R}$ factor thus quantifies the net reduction in transport induced by zonal flows relative to the bare gyro-Bohm level.

\section{IMPORTANCE OF ZONAL FLOWS FOR MAGNETIC CONFINEMENT FUSION RESEARCH}

One frequently hears the question or comment "zonal flows are interesting, but just why are they important for fusion?" There are at least five parts to the answer to this question, which are listed below.

First, zonal flows regulate drift wave turbulence and transport, thus reducing transport coefficients in comparison to the gyro-Bohm reference value. This reduction, quantified by the $\mathcal{R}$-factor, gives an intrinsic effective $H$-factor, even in the absence of a transport barrier. The impact of improved confinement on the fusion experimental reactor has been assessed that the necessary system size, $L$, for self-sustained burning is reduced by the $\mathrm{H}$-factor as $L \propto H^{-1.3 .}{ }^{8}$ Given that $H_{\text {eff }} \sim \mathcal{R}^{-0.6}$, it follows that the necessary current is reduced like $L \propto \mathcal{R}^{0.8}$ and zonal flow improvements can mitigate the cost of a reactor (where $\mathcal{R}$ can be much smaller than unity).

Second and in a similar vein, zonal flows can shift the effective boundary for the onset of significant turbulence and transport. This shift in onset, often referred to as the "Dimits shift," ${ }^{\prime \prime}$ is a consequence of the fact that close to instability threshold in low collisionality regimes, most of the available free energy is coupled directly to zonal flows, with comparatively little left for cross-field transport. This Dimits shift regime may be thought of as a limiting case of the regime of cyclic bursts, produced by the competition between shearing (predator) and fluctuation growth (prey). As the interburst period is set by the zonal flow damping, it becomes arbitrarily large in the limit of weak collisional damping. ${ }^{10}$ The onset of the Dimits shift regime may be thought of as a soft (i.e., second order) transition to an improved regime.

Third, and still continuing in the previous vein, the study of zonal flows has identified a new control knob on transport, which is the zonal flow damping rate. In the case of a tokamak, the damping in the low collisionality regime is set by the ion-ion collisionality, and the magnetic geometry, as it is due friction between trapped and untrapped particles. ${ }^{11,12}$ The scaling of zonal flow damping in other configurations, such as stellerators and spherical tori, is of great interest and has not been addressed much.

Fourth, the study of zonal flows has advanced our understanding of secondary mesoscale fluctuations and their effect on tokamak phenomena. These structures include zonal fields (i.e., the magnetic counterparts of zonal flows), which can seed neoclassical tearing modes, and avalanches, which trigger an intermittent heat flux and which necessarily compete against zonal flows. Thus, zonal flow physics enters considerations pertaining to the beta limit and peak heat loads. Zonal flow physics has also been linked to resistive wall mode dynamics.

Fifth, the study of zonal flow physics has led to a type of 
paradigm shift, so that self-generated shear layers are now viewed as an equal constituent, along with drift waves, in drift wave turbulence. This has sparked the development of the "predator-prey" paradigm of drift wave-zonal flow turbulence, discussed here and in the references.

\section{ZONAL FLOW PHYSICS-A CRITICAL ASSESSMENT}

We now proceed to a critical assessment of our understanding of zonal flow physics. This assessment divides the issues into three groups:

(a) what we understand,

(b) what we think we understand, but need more work on, and

(c) what we do not understand.

In this section, we discuss theoretical aspects of (a) and

(b) sequentially. The experimental observations are discussed in the next chapter. Then issue (c) is discussed in Sec. VI.

\section{A. What we understand about zonal flows}

It is clear that several aspects of zonal flows physics are now well understood theoretically. These are:

(1) Zonal flows are universal phenomena in microturbulence.

(2) In low collisionality regimes, zonal flows damp by scale-independent friction between trapped and circulating ions.

(3) Zonal flows are pumped by modulational instability of the drift wave gas. The detailed manifestation of the modulational instability dynamics varies considerably with parameters.

(4) Drift wave zonal flow turbulence is a self-regulating system, of the predator-prey type. Hence, both stationary and bursty or cyclic states are possible.

Assertions (1)-(4) are discussed in the following.

The universality of zonal flows is indisputable. Zonal flow generation and the concomitant shear suppression of turbulence and transport has been observed in numerical simulations of ITG, collisionless trapped electron mode, ETG, resistive ballooning, resistive interchange and collisional drift wave turbulence. This list encompasses virtually all of the candidates for the cause of anomalous transport.

Zonal flows damp (linearly) in low collisionality regimes by (collisional) friction between circulating ions and trapped ion bananas. For standard tokamak geometry, in the banana regime, $\gamma_{\mathrm{damp}} \cong-\nu_{i i} / \varepsilon_{T}$, so the damping is proportional to ion-ion collisionality. Note that the zero-frequency zonal flow is damped only by collisions. Hence, even in "nearly collisionless regimes," the zonal flow damping is strictly collisional.

It is now quite clear that flows grow by modulational instability. In all cases, a modulational stability analysis seeks to explore the stability of a drift wave state to a "test" zonal flow shear. The basic drift wave state can be one of a single coherent mode, or one of drift wave turbulence. The stability analysis can be linear, or, with additional strong simplifying assumptions, nonlinear. Thus, modulational sta-

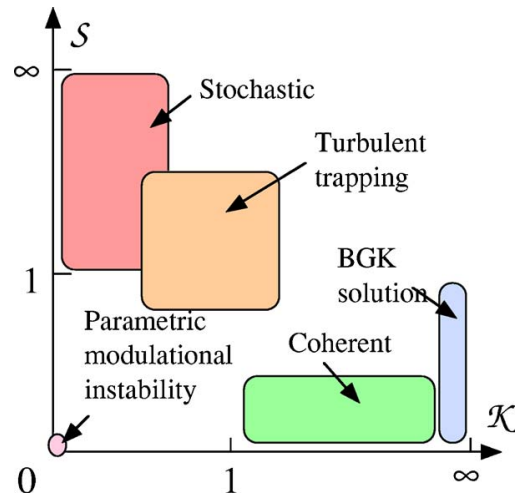

FIG. 3. Parameter domains for various theoretical approaches.

bility of drift waves has been predicted by single wave parametric modulational calculations, ${ }^{13,14}$ spectral wave-kinetic calculations, ${ }^{15-19}$ reductive perturbation theory analyses, ${ }^{20-22}$ and by studies of coherent $\mathrm{t}^{23-25}$ and turbulent ${ }^{26}$ trapping. The wide variety of approaches (i.e., see Chap. 3 of Ref. 1) to the modulational problem can be instructively classified in the 2D space of drift wave Kubo number and drift wave ray Chirikov parameter, as shown in Fig. 3. (The Kubo number is the ratio of the autocorrelation time of DW to the circumnavigation time of DW packet in the zonal flow field. The Chirikov parameter is the ratio between the trapping width in the group-velocity space of DW packet and the separation of phase velocities of multiple zonal flows.) Modulational instability of developed drift wave turbulence has been verified by a numerical experiment using gyrokinetic particle simulation codes. ${ }^{27}$ Thus, it seems incontrovertible that modulational instability of drift waves is the route to zonal flow formation.

\section{B. What we think we understand about zonal flows, but need more research on}

As mentioned earlier, zonal flow dynamics are naturally self-regulating. This follows from the fact that zonal flow generation extracts energy from drift waves, which may in turn be dissipated by zonal flow damping mechanisms. Note that fixed points, limit cycle states, and fixed points with long relaxation times (i.e., which are the time asymptotic states of slow modes) are possible.

Several other basic aspects of zonal flow physics are understood, but remain topics of current research in the hope of obtaining deeper or more precise quantitative understanding. These are:

(1) Several viable mechanisms for zonal flow saturation in regimes of low collisionality have been identified and explored.

(2) Zonal flows can modify the onset criterion for turbulence and turbulent transport.

(3) Magnetic field structures with $m=n=0$ and finite radial wave number, called zonal fields, can be generated by drift wave turbulence.

(4) The interaction of zonal and mean flows at the $\mathrm{L} \rightarrow \mathrm{H}$ transition can lead to pretransition dithering and modify hysteresis. 
(5) A variant of the zonal flow, referred to as the GAM (geodesic acoustic mode), is likely important in regulating edge turbulence.

(6) Zonal flow "control knobs" have been identified. Assertions (1)-(6) are discussed in the following.

At least three types of collisionless saturation mechanisms for zonal flows have been identified, any one of which can define an effective $\gamma_{\mathrm{NL}}\left(W_{\mathrm{ZF}}\right)$, which in turn yields a finite value of $W_{d}$ as $\gamma_{\text {damp }} \rightarrow 0$. These mechanisms include higher order scattering of drift wave rays in zonal flows, ${ }^{28}$ ray trapping in zonal flows, ${ }^{23,24}$ and Kelvin-Helmholtz (i.e., "tertiary") instability of the zonal flows. ${ }^{29}$ The higher order wave kinetics is very similar to nonlinear Landau damping, familiar from weak turbulence theory. Here the drift wave ray corresponds to the "particle" and the zonal flow corresponds to the "wave." Third order perturbation theory yields a nonlinear saturation effect, proportional to the mean square zonal flow shear. The reliability of the theory near drift wave marginality needs further investigation, though. Continuing in this vein, the trapping calculation may be viewed as a study of Bernstein-Greene-Kruskal solutions (of the wave kinetic equation), which trap drift wave rays. The condition of saturation ties the ray "bounce" frequency to the drift wave growth rate. Further study of this interesting mechanism is necessary in order to assess its viability in systems of practical interest. A third type of saturation mechanism is Kelvin-Helmholtz type instability of the zonal flows, driven by their shear. Such an instability would generate a turbulent viscosity, thus damping zonal flows and coupling some of their energy back to the drift wave spectrum. Although such tertiary instability is a natural candidate for zonal flow saturation, its stability in a sheared magnetic field, its dependence on zonal flow profile, and its behavior in a background of drift waves are all quite subtle, and much more research is required to quantitatively assess its viability as a zonal flow saturation mechanism. ${ }^{30}$ Although return of energy from the zonal flow repository to the drift waves gas is possible, it does not readily occur, especially in plasmas with magnetic shear.

As noted before, in low collisionality plasmas the extent of the Dimits shift regime defines an effective shift in the critical gradient for the onset of significant transport. This shift occurs in the region "near" marginal stability, thus begging the question of "just how near is near?" This is effectively the same question as the one pertaining to the mechanism of collisionless saturation. Thus, the physics of higher order wave kinetic processes, ray trapping and tertiary instability, all can define an effective shift. The quantitative impact of this on confinement is presently under study, as is the parameter dependence of the extent of the Dimits shift regime.

Zonal flow physics in finite beta plasmas exhibits two important new features. First, magnetic stresses grow and compete against $E \times B$ velocity-induced stresses, i.e., $\left\langle\widetilde{v}_{E, x} \widetilde{v}_{E, y}\right\rangle \rightarrow\left\langle\widetilde{v}_{E, x} v_{E, y}\right\rangle-\left\langle\widetilde{B}_{x} \widetilde{B}_{y}\right\rangle$, thus reducing or quenching the amplification of velocity shear. This will, in turn, usually result in a net increase in the turbulence level. A second effect is the amplification of secondary magnetic fields with a structure similar to that of zonal flows. ${ }^{31}$ These fields, called zonal fields, can react back on the turbulence via a process of random or corrugated magnetic shearing. The process of zonal field amplification may be thought of as one where the turbulence produces a current layer via a "negative turbulent resistivity," generated by drift Alfvén wave spectra with slopes of a certain sign. The generation process proceeds via transport of magnetic potential, just as the zonal flow generation process proceeds via vorticity transport. It should be noted that although this process amplifies field, it does not amplify magnetic flux, and so is not, strictly speaking, a "dynamo" in the conventional sense of the word ${ }^{32}$ (i.e., as in the alpha-effect). Rather it is a process which redistributes flux, causing local field intensification. Nonlinear simulations have shown generation of zonal fields and large scale magnetic field. ${ }^{33-35}$ In practice, zonal field magnetic shearing is usually weak, for most tokamak parameter regimes. As with zonal flows, some possibility of tertiary instability of zonal fields exists as well. In this case, the most likely instability mechanism would be something akin to a current and electron temperature gradient driven microtearing instability. One other interesting offshoot of zonal field research is the realization that zonal fields may seed neoclassical tearing modes. $^{36}$

Zonal flows differ from the mean $\mathbf{E} \times \mathbf{B}$ flow, in that the latter can persist in the absence of turbulence, whereas the former are exclusively turbulence driven. Mean flows can thus suppress zonal flows, as well as drift waves, by quenching the turbulence which drives the zonal flows. As a consequence, there are interesting possibilities for energy exchange between the zonal flows and the mean flow. A simple model has been used to explore this possibility, and has predicted that zonal flow coupling will produce pretransition dithering, modify the expected transition threshold and alter the backtransition hysteresis. ${ }^{37}$ Further work is needed, especially on the implications of zonal flows for the dynamics of pedestal buildup.

Two practical points concern the relation between zonal flows and GAMs, and the possibility of using aspects of zonal flow physics as to construct a "control knob" for the turbulent transport. First, at lower temperatures characteristic of the edge $\nu_{i i}$ increases and $c_{s} / R$ decreases, so the characteristic frequencies of zonal flows and GAMs begin to overlap. ${ }^{38}$ As a consequence, geodesic-acoustic coupling becomes an important element in, and limitation on, zonal flow generation processes. ${ }^{39}$ Second, an understanding of the zonal flow drive and damping has suggested several routes to improving confinement via externally driven flow shear amplification $^{40,41}$ or by tuning the configuration design to lower the zonal flow damping. Effects of helical geometry have been studied in Refs. 42 and 43. These provide opportunities to test the theory, as well as possibilities for optimizing the influence of zonal flows, through examining the dependence of the damping on helical geometries.

Before closing this section, a few notes are addressed to the universality of zonal flows. In studying the electron transport, caused by ETG or trapped electron modes (TEM), the role of ZFs has been a subject of active discussion. In the dynamics of ETG and ZFs, a near isomorphism holds be- 
tween the system of DW-ITG and ZFs. As is discussed in Sec. II A, the growth of ZFs in ETG turbulence can be slower than the case in ITG turbulence. Intensive efforts have been made to determine the level of ZFs, characteristic radial scale lengths of the turbulence, and the turbulent energy flux in stationary state. These are of issues in current research, surveyed in Refs. 1 and 2, and further discussion is given in Refs. 44-47. Another current topic is the study of ZFs in TEM turbulence. For this current research subject, readers are referred to the review and references therein along with Refs. $48-51$ for a more detailed discussion of the latest results.

\section{EXPERIMENTAL STUDIES}

Recent years have witnessed very rapid progress in experimental studies of zonal flows. Here, we write "low frequency zonal flows (LFZFs)" to discriminate between the quasi-zero-frequency zonal flows and the GAMs. Issues which must be addressed are:

(1) the identification of zonal flows (LFZFs and GAMs);

(2) spatiotemporal structures (frequency, radial wave number, coupling with $\mathrm{m}=1$ component);

(3) generation of flow shear (or torque) by turbulence;

(4) the nonlinear coupling coefficient between ZFs and microturbulence; and

(5) the suppression of turbulent fluctuations by ZFs.

\section{A. Identification of ZFs and spatiotemporal structure}

The most important criteria for identifying zonal flows is the homogeneity of the potential perturbation over a magnetic surface and the rapid variation of the potential across magnetic surfaces. Simultaneous measurement of fluctuations at different positions, the distance between which is longer than the scale length of the microfluctuations, has been made using heavy ion beam probe (HIBP) ${ }_{52,53}^{5}$ beam emission spectroscopy, ${ }^{54}$ Langmuir probes $^{55-57}$ and reflectometry. ${ }^{58,59}$ These diagnostics allow us to observe ZFs, and coherent fluctuations of electrostatic potential, the poloidal mode number of which is estimated to be zero. Low frequency zonal flows and GAMs are now widely observed, on many devices. (Note that the property of $n=0$ is confirmed only in limited experiments. In many tokamak experiments, $n=0$ is attributed when $m=0$ is confirmed, based on the high electric conductivity in hot plasmas.)

\section{Low frequency ZFs}

It has been shown that LFZFs really exist in toroidal plasmas. Confirmation of $m=n=0$ for LFZFs has been achieved by dual-HIBP measurement of electric field on compact helical system (CHS). ${ }^{52,53}$ (See Fig. 4.) The radial wavelength, which was measured by observing the crosscoherence between the radial electric field at two radii, was found to be about 20 ion gyroradius. This value is within the range of theoretical predictions. An accompanying density fluctuation is very weak, and is within the experimental error bar. The LF zonal flows are observed in all regions of the plasma column and in the L-mode and in the internal trans-

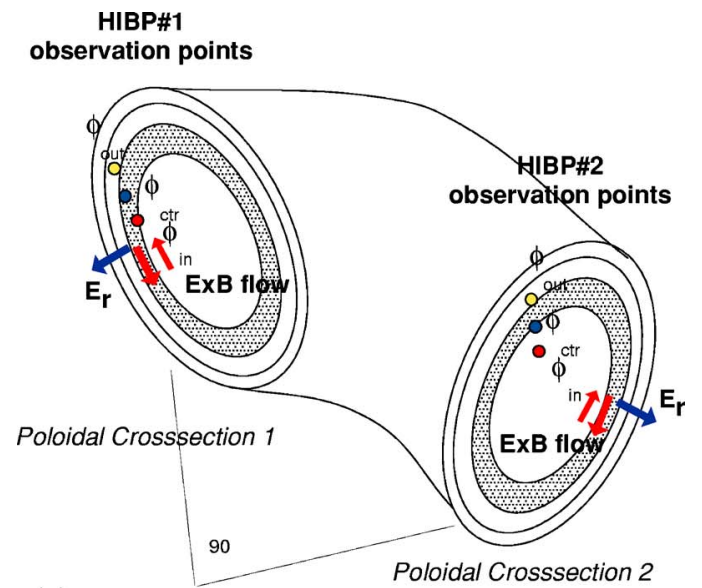

(a)

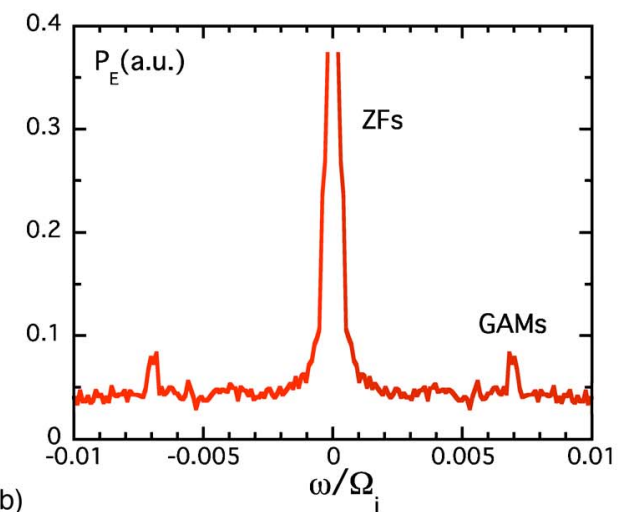

(b)

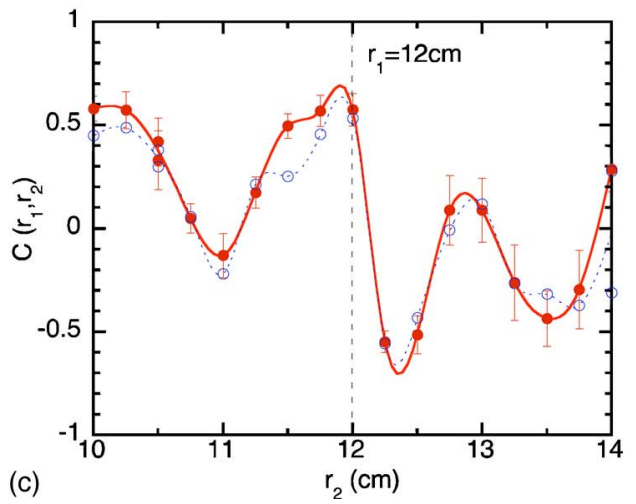

FIG. 4. Identification of low frequency zonal flows on CHS. (a) Geometry for measurement is schematically shown. (b) Power spectrum, in which a peak of LFZFs and of GAMs are observed. (c) Cross-correlation function of low frequency fluctuations measured by HIBP-1 and HIBP-2 are calculated, and its radial dependence. In this case, the radius of measurement by HIBP-1 is fixed as $r_{1}=12 \mathrm{~cm}$, and the observation radius of HIBP-2, $r_{2}$, is varied. (Quoted from Refs. 52 and 53.)

port barrier. It was found that the zonal flow amplitude is stronger on inside of the internal transport barrier of CHS plasma. This is related to the fact that the inside of internal transport barrier, which is realized by electron cyclotron heating on CHS, is characterized by a strong positive radial electric field (electron-root branch). The damping of the radial electric field, which deviates from the neoclassical charge neutrality condition, is weaker in the electron-root plasma in comparison with the one in the ion-root. Thus, for a given driving torque, ZFs can be stronger in the electronroot plasma, in the case of CHS. LFZFs were observed to 


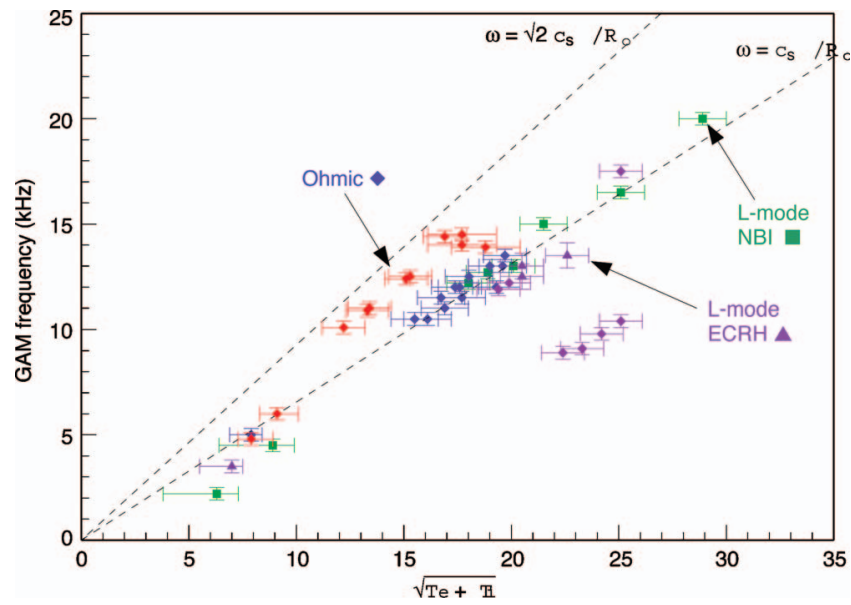

FIG. 5. (Color) Dependence on the temperature of the peak frequency, which is attributed as GAMs. (Result from ASDEX-U, quoted from Ref. 58.)

oscillate in time when measured at one location. The origin of this oscillation may be (i) radial propagation of ZFs or (ii) dynamics induced by coupling with turbulence (e.g., predator-prey-like dynamics or trapping of turbulence, etc.), but the origin of the oscillations has not yet been clarified. That is, the finding of LFZFs has opened a future full experimental studies of zonal flow physics. Emerging evidence is has also been reported. ${ }^{1,58,60}$

\section{GAMs}

Many observations of GAMs have been reported. When fluctuations of electric field, flow velocity and density are measured, a peak in spectrum appears near the GAM frequency at the location of the measurement. ${ }^{1}$ Although the toroidal mode number has not yet been definitively measured in many tori, a long poloidal correlation length (not contradicting $m=0$ ) is considered to be a strong reason to accept these peaks are due to GAM oscillations. A correspondence to the GAM frequency has been studied widely to test linear theory. An example, quoted from Ref. 58, has shown that the measured frequency (for fixed measurement domain) is close to the local GAM frequency. (See Fig. 5.) The dependence on the plasma parameters, such as the safety factor, was also reported. $^{61}$

Radial structure has been studied, ${ }^{54,58,62-64}$ and the radial propagation of the wave was identified. The radial wave length was found to be order of ten ion-gyroradii. (See the result of JFT-2M, ${ }^{64}$ Fig. 6.) In addition, the relation between the density perturbation amplitude $(\tilde{n} / n)$ and electric potential perturbation $e \widetilde{\phi} / T_{e}$ was studied. The phase and magnitude of $\tilde{n} / n$, relative to $e \widetilde{\phi} / T_{e}$, confirmed the observation of the linear eigenmode features of the GAM. More explicit confirmation of the $\sin \theta$ dependence of $\tilde{n} / n$ must be performed in future.

\section{B. Generation of flow shear (torque) by turbulence}

The fluctuation-driven Reynolds stress has been measured in various devices. Fluctuation velocities (in the radial as well as poloidal directions, $\widetilde{v}_{r}$ and $\tilde{v}_{\theta}$ ) are measured, and a

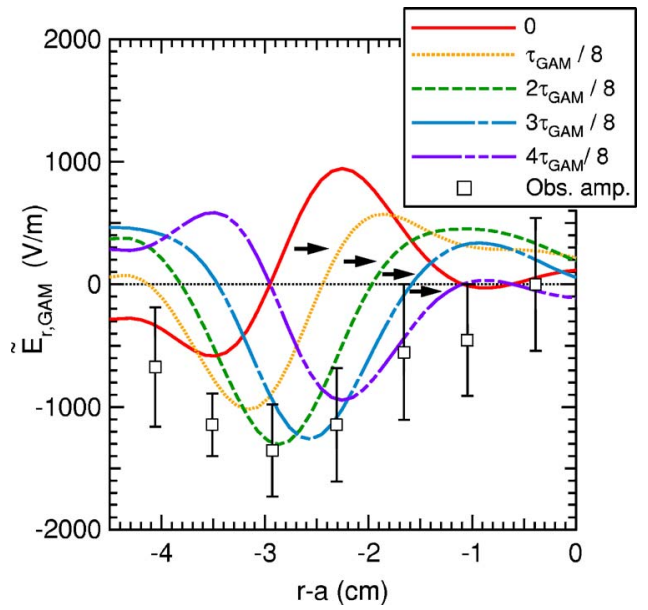

FIG. 6. Observation of radial propagation of GAMs, measured by HIBP on JFT-2M plasma. (Quoted from Ref. 64.)

time average stress $\Pi_{r \theta}=\left\langle\widetilde{v}_{r} \widetilde{v}_{\theta}\right\rangle$ has been evaluated. One must be careful in interpreting the result, because the measurement of $\left\langle\widetilde{v}_{r} \tilde{v}_{\theta}\right\rangle$ is often limited to one point in space, and the average over the magnetic surface replaced by the time average. Although this limitation demands further experiments for quantitative conclusion, present observations have yielded significant understanding. Perhaps the most detailed comparison has been reported in basic plasma experiments on linear plasmas. By taking the radial derivative of $\Pi_{r \theta}$, the torque in the poloidal direction was estimated. Using the neutral particle drag, calculated by use of the measured neutral profile, one can estimate the plasma flow velocity in the poloidal direction. This estimated flow was compared to the direct measurement of the flow. Reference 65 reports semiquantitative agreement, showing that the turbulence-driven stress is the dominant element of the drive for the global plasma flow shear. (See Fig. 7.) Observation on a large tokamak was reported in Ref. 66.

As was explained in the review, ${ }^{1,2}$ the generation of the shear by the convection motion driven by instabilities is associated with the tilt of the convection pattern. Such a tilt of the convection pattern has been observed in detail in linear plasma experiments. ${ }^{55,67}$ In toroidal plasmas, the tilt of convection instability has also been observed. ${ }^{57}$

In the studies reported in Refs. 68 and 69, the contribution of the Maxwell stress is also discussed together with the Reynolds stress.

\section{Nonlinear coupling coefficient between ZFs and microturbulence}

\section{Bicoherence analysis}

A direct experimental confirmation for the nonlinear interaction among three waves is given by use of the bicoherence analysis. Consider data in a form of a time series $y(t)$. The bicoherence indicator is the three time correlation, ${ }^{70}$ and is evaluated by use of the Fourier spectrum $Y(\omega)$ as

$$
B\left(\omega_{1}, \omega_{2}\right)=\left\langle Y^{*}\left(\omega_{1}\right) Y\left(\omega_{2}\right) Y\left(\omega_{1}-\omega_{2}\right)\right\rangle,
$$

where the angular brackets $\langle\cdots\rangle$ indicate a statistical average over a large number of realizations. The fluctuation spectrum 

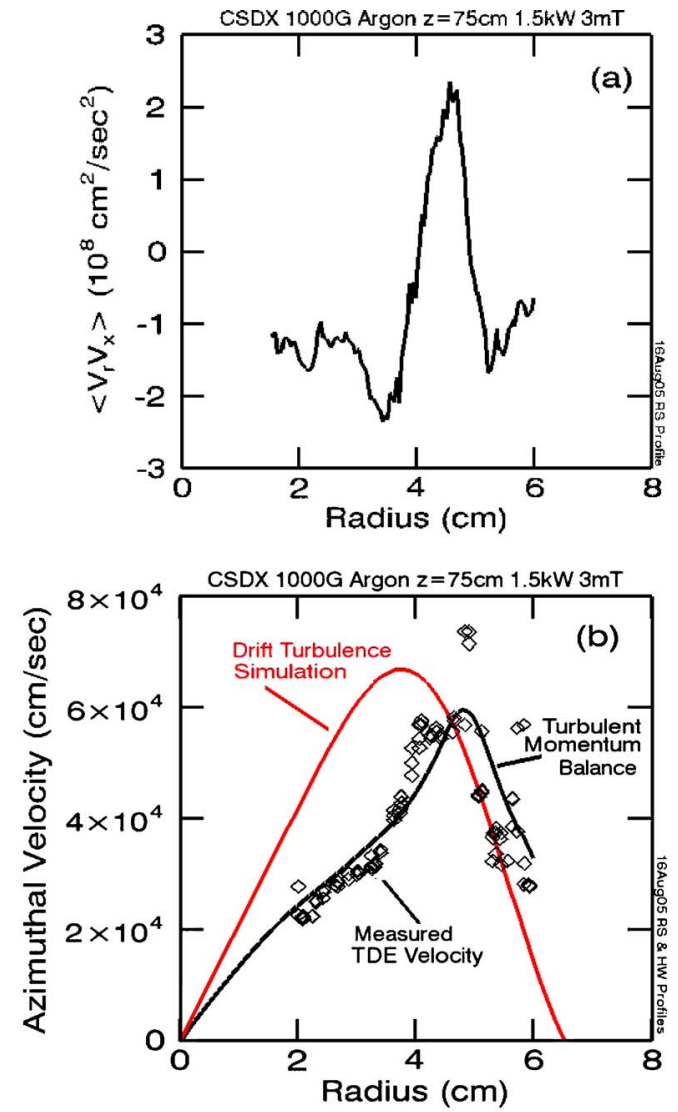

FIG. 7. Radial profile of (a) the turbulence-driven Reynolds stress and (b) the poloidal flow in the CSDX experiment. In (b), the plasma flow velocity is compared to the estimation given by balancing the turbulent Reynolds stress and drag on ions. Prediction of turbulence simulation is also illustrated. (Quoted from Ref. 65.)

is given, in general, by the space-time Fourier decomposition, e.g., a power spectrum $I(k, \omega)$. However, a complete spatiotemporal data set is not usually obtained by experiments. The bicoherence analysis of experimental data has often been performed on the temporal Fourier spectrum. Such studies are relevant to cases where the condition of the wave number matching $\mathbf{k}_{1}=\mathbf{k}_{2}+\mathbf{k}_{3}$ is (approximately) satisfied if the frequency matching condition $\omega_{1}=\omega_{2}+\omega_{3}$ is fulfilled. The interactions between drift waves and zonal flows belong to this class of interaction. The total bicoherence $\sum \hat{b}^{2}(\omega)$ has been theoretically analyzed. It was shown that $\sum \hat{b}^{2}(\omega)$ at the frequency of the zonal flows (LFZFs and GAMs) is proportional to the amplitude of the ZFs. ${ }^{71,72} \mathrm{An}$ explicit formula was derived in Ref. 72. A detailed study was performed on JFT-2M plasmas. ${ }^{73}$ It was shown that $\Sigma \hat{b}^{2}(\omega)$ at the GAM frequency increases in proportion to the ratio $\widetilde{\phi}_{\mathrm{ZF}}^{2} / \widetilde{\phi}_{d}^{2}\left(\widetilde{\phi}_{d}\right.$ is a potential fluctuation amplitude for drift waves) confirming theories.

Another important measurement is the biphase. It was shown that the biphase $\Theta$, defined as $B\left(\omega_{2}, \omega_{2}\right)$ $=\exp (i \Theta)\left|B\left(\omega_{1}, \omega_{2}\right)\right|$, reveals another feature of the nonlinear interactions. Biphase analysis of edge turbulence of JFT-2M has shown that $\Theta\left(\omega_{1}, \omega_{2}\right)$ for GAMs (i.e., $\left.\omega_{1}+\omega_{2}=\omega_{\mathrm{GAMs}}\right)$ has weak dependence on $\omega_{1}$, confirming that ambient microfluctuations are coherently modulated by GAMs. ${ }^{74}$

\section{Energy transfer between fluctuations}

The evolution equation for spectral power $P_{k}=\left|\phi_{k}\right|^{2}$ may be written as

$$
\frac{\partial}{\partial t} P_{k}=\gamma_{k} P_{k}+\sum_{k_{1}}^{\prime} T_{k}\left(k_{1}, k_{2}\right),
$$

where $T_{k}\left(k_{1}, k_{2}\right)$ is the transfer function of energy from the modes $k_{1}$ and $k_{2}$, and the summation $\Sigma^{\prime}$ means the sum over $k_{1}$ with the constraint $k=k_{1}+k_{2}$. The coefficient $T_{k}\left(k_{1}, k_{2}\right)$ is given by the third order moment of a fluctuation quantity, multiplied by the nonlinear coupling coefficient. The energy density associated with the potential perturbation is given as $E_{k}=\left(1+k_{\perp}^{2} \rho_{s}^{2}\right)\left|\phi_{k}\right|^{2}$, so that the energy transfer function is rewritten as $W_{k}^{\mathrm{tr}}=\left(1+k_{\perp}^{2} \rho_{s}^{2}\right) \Sigma_{k_{1}}^{\prime} T_{k}\left(k_{1}, k_{2}\right)$. By measuring the statistically averaged values of the second, third, and fourth order moments, the growth/damping rate $\gamma_{k}$ and the energy transfer function $W_{k}^{\mathrm{tr}}$ are evaluated. ${ }^{75}$ Recent experimental efforts have been reported in Ref. 76. It turned out that $W_{k}^{\mathrm{tr}}$ is positive, i.e., energy is nonlinearly pumped into the low frequency component, and that $W_{k}^{\mathrm{tr}}$ is negative (i.e., the energy is nonlinearly damped) in the high frequency region. This result is consistent with the theoretical expectation that GAMs (which are observed around $\omega / 2 \pi \sim 6 \mathrm{kHz}$ in this case) are excited by high frequency microfluctuations. Further detailed studies are necessary.

\section{Envelope modulation}

Other way to identify the nonlinear interaction between ZFs and microfluctuations is to observe the amplitude modulation of the envelope of microscopic fluctuations. As is explained in the theory of zonal flow growth based on the modulational instability, the excitation of zonal flows is accompanied by the envelope modulation of background microfluctuations. This approach has been used in various experiments. ${ }^{62,64,76,77}$

One example is quoted from observations on the JFT-2M tokamak. ${ }^{64}$ The high frequency part is taken from the potential fluctuation of the edge turbulence $\widetilde{\phi}$ (HIBP measurement). The high-pass filter is used $(\omega / 2 \pi>60 \mathrm{kHz})$, and the envelope of this filtered signal, $a(t)$, is calculated by Hilbert transformation. The envelope signal $a(t)$ is analyzed. There is a clear correlation between the envelope and the GAM oscillation which appears around $\omega / 2 \pi=16 \mathrm{kHz}$. This correlation of the envelope $a(t)$ and the GAM oscillation are compared with theoretical predictions. In the experiments, the relative magnitude of the envelope modulation $a(t)$ [normalized to $\left.\sqrt{\left\langle|\phi|^{2}(\omega / 2 \pi>60 \mathrm{kHz})\right\rangle}\right]$ at the frequency of GAMs is found to be around $20 \%$. By use of the observed parameters of the plasma, GAMs and microfluctuations, a theoretical model $^{78}$ has predicted that the relative modulation amplitude at the location of measurement is about $23 \%$. A semiquantitative confirmation of this prediction has been obtained. ${ }^{64}$

\section{Suppression of turbulent fluctuations by ZFs}

The low-frequency zonal flows are considered to be more effective than GAMs in suppressing the turbulence. 


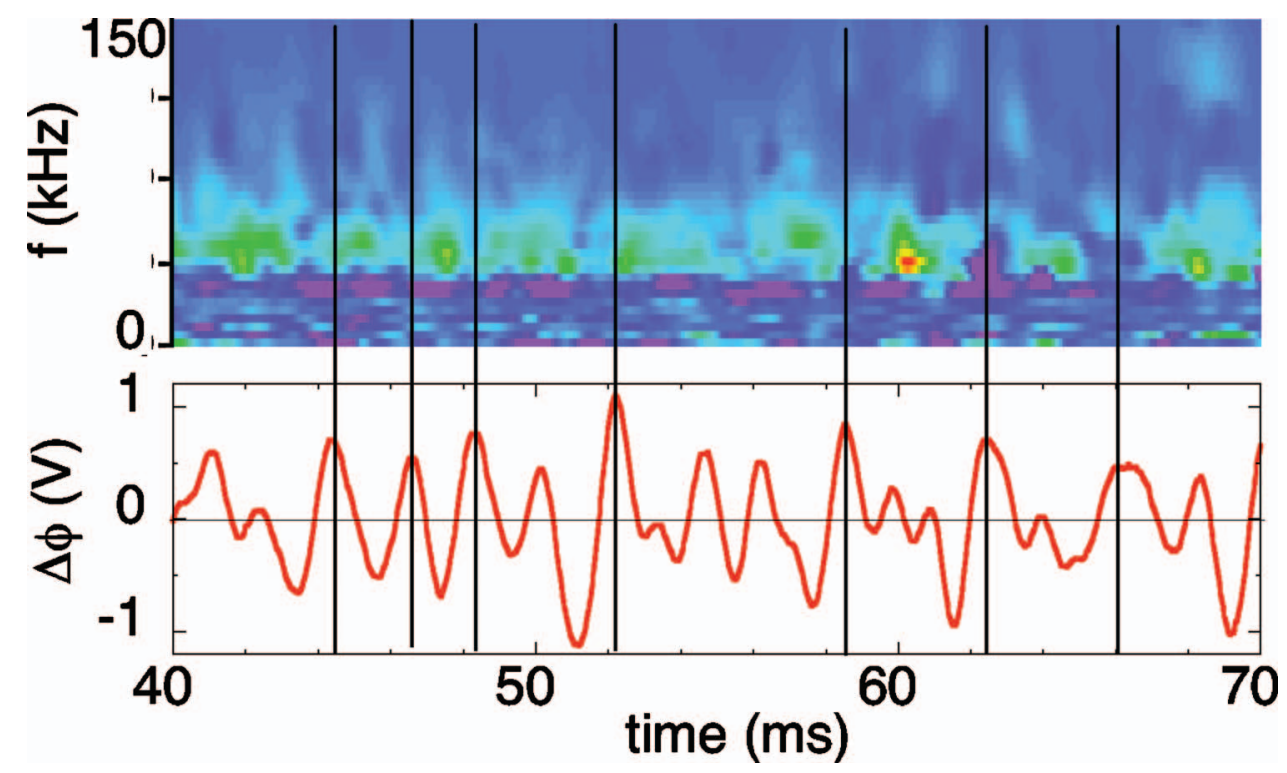

FIG. 8. (Color) Effect of LF zonal flows on turbulent transport. Temporal evolution of spectral function $\Gamma(\omega)$ is plotted with the electric field variation of the zonal flow. (Result from CHS, quoted from Ref. 79.)

Thus, the identification of turbulence suppression by LF zonal flows is the focus of experimental studies of zonal flows.

Measurement of the ZFs effects on turbulent transport has been made on CHS. ${ }^{79}$ The HIBP measurement provides local data of the electric field perturbation $\widetilde{E}_{\omega}$, the density perturbation $\widetilde{n}_{\omega}$, and the phase difference between them $\alpha_{\omega}$. The particle flux is measured as

$$
\begin{aligned}
& \Gamma=\sum_{\omega} \Gamma_{\omega}, \\
& \Gamma_{\omega}=\frac{1}{B}\left\langle\widetilde{E}_{\omega} \widetilde{n}_{\omega} \cos \alpha_{\omega}\right\rangle .
\end{aligned}
$$

It was found that $\Gamma_{\omega}$ (carried by high frequency fluctuations, $\omega>40 \mathrm{kHz}$ ) is modulated by the LFZFs. $\Gamma_{\omega}$ is high at the bottom of the zonal flow plot (where the ZF electric field is opposite to the mean dc radial electric field) and low at the peak of the zonal flow plot (where the ZF electric field is the same as the mean dc radial electric field) in the plasmas which fall in the electron-root branch. (See Fig. 8.) This observation is consistent with theoretical modeling, which predicts that fluctuations tend to accumulate in the trough of ZFs.

Observations have also been reported on the interaction of GAM oscillations and turbulent transport. A characteristic example is the HIBP measurement of the edge turbulence on the JFT-2M tokamak. ${ }^{62}$ The particle flux $\Gamma=\Sigma_{\omega} \Gamma_{\omega}$ was evaluated based on HIBP data. It was found that $\Gamma$ is modulated at the frequency of the observed GAM oscillations. The time series data $\Gamma(t)$ is analyzed, and its Fourier spectrum $\Gamma(\omega)$ was calculated. The probability density function (pdf) of $\Gamma(\omega)$ is illustrated in Fig. 9. It is clear that the particle flux $\Gamma(t)$ is modulated at the GAM frequency.

\section{FUTURE RESEARCH-WHAT WE DO NOT YET UNDERSTAND ABOUT ZONAL FLOW PHYSICS}

After surveying progresses in theory, simulation and experiments, we briefly comment on future task. There are several aspects of zonal flow physics where significant future study is required. These are as follows:

(1) Other convincing experimental links between zonal flow excitation and enhancement of confinement need to be established.

(2) The rule governing how the system selects among the various collisionless saturation mechanisms must be derived and validated.

(3) Quantitative predictability (with understanding) remains elusive. In particular, quantitative algorithms for actually calculating the $\mathcal{R}$-factor, the extent of the Dimits shift regime, the threshold for shear suppression and the onset of bifurcative transitions must all be developed.

(4) Our understanding of the interaction and competition between various secondary structures, such as zonal flows

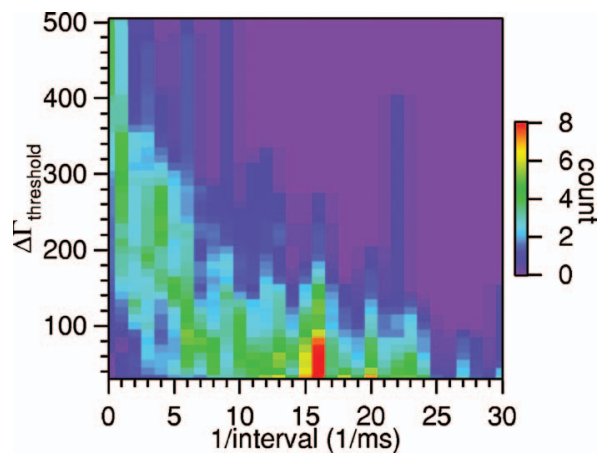

FIG. 9. (Color) The pdf of modulation of the particle flux, $\Delta \Gamma$, at various frequency. There is a clear peak at the frequency of GAMs $(\omega / 2 \pi$ $\sim 16 \mathrm{kHz}$ in this case). (Result from JFT-2M, quoted from Ref. 62.) 
and avalanches or streamers, is rudimentary and must be improved.

(5) The factors limiting the efficiency of the control of zonal flows must be determined.

(6) Further development of statistical theory for the system of drift wave and zonal flows (for which readers are referred to Refs. 1, 2, 17, 26, 80, and 81) are necessary to address the above-listed issues.

Clearly, many interesting challenges and discoveries await researchers working on the subject of zonal flows.

\section{CONCLUSION}

In conclusion, the study of zonal flow physics had led to a paradigm shift in our most basic concept of "drift wave turbulence and transport." We now think of "drift wave-zonal flow turbulence" as a self-regulating system, with shearing and shear-induced modulational instability providing the key feedback elements. This natural self-regulation can be important for magnetic fusion, both by defining a reduction in transport relative to gyro-Bohm (i.e., the $\mathcal{R}$-factor, discussed earlier) and by its effect on transport barrier formation. Very rapid progress has been made these years concerning on the experimental studies of zonal flows. We can look forward with confidence to future discoveries of the role of secondary flows and fields in different areas of fusion physics, such as toroidal Alfvén eigenmode (TAE) instabilities, neoclassical tearing modes and resistive well modes, just to name a few possibilities. The progress of plasma physics induced a paradigm shift form the previous "linear, local and deterministic" view of turbulent transport to the new "nonlinear, nonlocal (in real and wave number spaces), statistical" view of turbulent transport. Physics of the drift wave-zonal flow system is a prototypical example of this evolution in understanding the turbulence and structure formation in plasmas.

It should also be noted that the zonal flows belong to a family of the problem that a large-scale (or mesoscale) axial vector field is generated from the large-scale inhomogeneity of the scalar field (such as the pressure, temperature, gravitational potential, etc.). ${ }^{82,83}$ Common physics can also be found in the dense plasma which is realized in conjunction with inertial fusion research. ${ }^{84}$ The dynamo problem and planetary and astrozonal flows also belong to this class. The process of global axial vector field generation via turbulence is the key for understanding the structure formation in plasmas far from equilibrium (in laboratory, as well as astrophysical circumstance). The study of zonal flows in laboratory plasmas provides the most detailed study to challenge this fundamental issue for understanding the evolution of plasmas in the universe.

\section{ACKNOWLEDGEMENTS}

This review has been performed with the help of numerous collaborators worldwide, names of which are listed in Ref. 1. The authors are grateful to Dr. Hallatschek and Dr. T. Ido for useful discussions and the authors of papers in Plasma Phys. Contr. Fusion cluster on "Experiments on zonal flows and turbulence," who have given us access to their unpublished work.

This research was supported by the Grant-in-Aid for Specially Promoted Research (16002005) of Ministry of Education, Culture, Sports, Science and Technology of Japan, by the Grant-in-Aid for Scientific Research (15360495), by the Collaboration Programs of NIFS (NIFS03KDAD001, NIFS03KKMD001) and the RIAM of Kyushu University, by Asada Eiichi Research Foundation, by the 4-2-4 programme of Kyushu University, by the U.S. DOE under Grant Nos. FG03-88ER53275 and FG02-04ER54738, and by the U.S. DOE Contract No. DE-AC02-76-CHO-3073. Some of the authors (K.I., P.H.D., T.S.H., A.F., G.R.T.) would like to acknowledge the hospitality of and financial support from Kyushu University, where much of this work was carried out. Another author (P.H.D.) also acknowledges support from and the hospitality of the Isaac Newton Institute for Mathematical Sciences, of the University of Cambridge.

${ }^{1}$ P. H. Diamond, S.-I. Itoh, K. Itoh, and T. S. Hahm, Plasma Phys. Controlled Fusion 47, R35 (2004).

${ }^{2}$ P. H. Diamond, S.-I. Itoh, K. Itoh, and T. S. Hahm, NIFS-805 (2004).

${ }^{3}$ P. H. Diamond, K. Itoh, S.-I. Itoh, and T. S. Hahm, Research Report NIFS-805 (National Inst. Fusion Science, Toki, 2004).

${ }^{4}$ S.-I. Itoh and K. Itoh, Plasma Phys. Controlled Fusion 43, 1055 (2001).

${ }^{5}$ C. Holland and P. H. Diamond, Phys. Plasmas 11, 1043 (2004).

${ }^{6}$ O. D. Gurcan and P. H. Diamond, Phys. Plasmas 11, 572, (2004); 11, 4973 (2004)

${ }^{7}$ Z. Lin, T. S. Hahm, W. W. Lee, W. M. Tang, and P. H. Diamond, Phys. Rev. Lett. 83, 3645 (1999).

${ }^{8}$ K. Itoh, S.-I. Itoh, and A. Fukuyama, Fusion Eng. Des. 15, 297 (1992).

${ }^{9}$ A. M. Dimits, G. Bateman, M. A. Beer, B. I. Cohen, W. Dorland, G. W. Hammett, C. Kim, J. E. Kinsey, M. Kotschenreuther, A. H. Kritz, L. L. Lao, J. Mandrekas, W. M. Nevins, S. E. Parker, A. J. Redd, D. E. Shumaker, R. Sydora, and J. Weiland, Phys. Plasmas 7, 969 (2000).

${ }^{10}$ M. A. Malkov, P. H. Diamond, and M. N. Rosenbluth, Phys. Plasmas 9, 5073 (2001).

${ }^{11}$ M. N. Rosenbluth and F. L. Hinton, Phys. Rev. Lett. 80, 724 (1998).

${ }^{12}$ F. L. Hinton and M. N. Rosenbluth, Plasma Phys. Controlled Fusion 41, A653 (1999).

${ }^{13}$ A. Hasegawa, C. G. Maclennan, and Y. Kodama, Phys. Fluids 22, 2122 (1979).

${ }^{14}$ L. Chen, Z. Lin, and R. B. White, Phys. Plasmas 7, 3129 (2000).

${ }^{15}$ R. Z. Sagdeev, V. D. Shapiro, and V. I. Shevchenko, Sov. J. Plasma Phys. 4, 306 (1978) [Fiz. Plazmy 4, 551 (1978)].

${ }^{16}$ A. Balk, S. V. Nazarenko, and V. E. Zakharov, Sov. Phys. JETP 71, 249 (1990).

${ }^{17}$ P. H. Diamond, M. N. Rosenbluth, F. L. Hinton, M. Malkov, J. Fleisher, and A. Smolyakov, Plasma Physics Controlled Nuclear Fusion Research (IAEA, Vienna, 1998), IAEA-CN-69/TH3/1.

${ }^{18}$ A. Yoshizawa, S.-I. Itoh, and K. I. Itoh, Plasma and Fluid Turbulence (IOP, Bristol, England, 2002).

${ }^{19}$ A. Smolyakov and P. H. Diamond, Phys. Plasmas 7, 1349 (2000).

${ }^{20}$ Y. Kodama and T. Taniuti, J. Phys. Soc. Jpn. 47, 1706 (1979).

${ }^{21}$ H. Sanuki and J. Weiland, J. Plasma Phys. 23, 29 (1980).

${ }^{22}$ S. Champeaux and P. H. Diamond, Phys. Lett. A 288, 214 (2001).

${ }^{23}$ P. K. Kaw, R. Singh, and P. H. Diamond, Plasma Phys. Controlled Fusion 44, 51 (2002).

${ }^{24}$ A. Smolyakov, P. H. Diamond, and M. Malkov, Phys. Rev. Lett. 84, 491 (2000).

${ }^{25}$ V. Marchenko, Phys. Rev. Lett. 89, 185002 (2002).

${ }^{26}$ R. Baleascu, Phys. Rev. E 68, 046409 (2003).

${ }^{27}$ P. H. Diamond, S. Champeaux, M. Malkov, A. Das, I. Gruzinov, M. N. Rosenbluth, C. Holland, and B. Wecht, Nucl. Fusion 41, 1067 (2001).

${ }^{28}$ K. Itoh, K. Hallatschek, S.-I. Itoh, P. H. Diamond, and S. Toda, Phys. Plasmas 12, 062303 (2005).

${ }^{29}$ B. N. Rogers, W. Dorland, and M. Kotschenreuther, Phys. Rev. Lett. 85, 5336 (2000).

${ }^{30}$ E. Kim and P. H. Diamond, Phys. Plasmas 9, 4530 (2002). 
${ }^{31}$ I. Gruzinov, A. Das, P. H. Diamond, and A. Smolyakov, Phys. Lett. A 302, 119 (2002).

${ }^{32}$ H. K. Moffatt, Magnetic Field Generation in Electrically Conducting Fluids (Cambridge University Press, New York, 1978).

${ }^{33}$ S. E. Parker, Y. Chen, W. Wan, B. I. Cohen, and W. M. Nevins, Phys. Plasmas 11, 2594 (2004).

${ }^{34}$ R. E. Waltz, J. Candy, F. L. Hinton, C. Estrada-Mila, and J. E. Kinsey, Nucl. Fusion 45, 741 (2005).

${ }^{35}$ M. Yagi, M. S.Yoshida, S.-I. Itoh, H. Naitou, H. Nagahara, J.-N. Leboeuf, K. Itoh, T. Matsumoto, S. Tokuda, and M. Azumi, Nucl. Fusion 45, 900 (2005).

${ }^{36}$ S.-I. Itoh, K. Ioth, and S. Toda, Phys. Rev. Lett. 89, 215001 (2002).

${ }^{37}$ E. Kim and P. H. Diamond, Phys. Plasmas 9, 4530 (2002).

${ }^{38}$ K. Hallatscheck, Phys. Rev. Lett. 84, 5145 (2000).

${ }^{39}$ B. D. Scott, Phys. Lett. A 320, 53 (2003).

${ }^{40}$ G. G. Craddock and P. H. Diamond, Phys. Rev. Lett. 67, 1535 (1991).

${ }^{41}$ T. Ohkawa and R. L. Miller, Phys. Plasmas 12, 094506 (2005).

${ }^{42}$ H. Sugama and T.-H. Watanabe, Phys. Rev. Lett. 94, 115001 (2005); Phys. Plasmas 13, 012501 (2006).

${ }^{43}$ T. Watari, H. Hamada, A. Fujisawa, K. Toi, and K. Itoh, Phys. Plasmas 12, 062304 (2005).

${ }^{44}$ F. Jenko, J. Plasma Fusion Res. 6, 11 (2004).

${ }^{45}$ Z. Lin, L. Chen, and F. Zonca, Phys. Plasmas 12, 056125 (2005).

${ }^{46}$ L. Chen, Z. Lin, and F. Zonca, "Nonlinear toroidal mode coupling: new paradigm for drift turbulence in toroidal plasmas," presented at 19th International Conference on the Numerical Simulation of Plasmas, Nara, 2005, Paper A5-3.

${ }^{47}$ W. M. Nevins, G. W. Hammett, A. M. Dimits, W. Dorland, and D. E. Shumaker, Phys. Plasmas 12, 122305 (2005).

${ }^{48}$ D. R. Ernst, P. T. Bonoli, P. J. Catto, W. Dorland, C. L. Fiore, R. S. Granetz, M. Greenwald, A. E. Hubbard, M. Porkolab, M. H. Redi, J. E. Rice, K. Zhurovich, and the Alcator C-Mod Group, Phys. Plasmas 11, 2637 (2004).

${ }^{49}$ K. Hallatschek and W. Dorland, Phys. Rev. Lett. 95, 055002 (2005).

${ }^{50}$ T. Dannert and F. Jenko, Phys. Plasmas 12, 072309 (2005).

${ }^{51}$ M. H. Redi, W. Dorland, C. L. Fiore, J. A. Baumgaertel, E. M. Belli, T. S. Hahm, G. W. Hammett, and G. Rewoldt, Phys. Plasmas 12, 072519 (2005).

${ }^{52}$ A. Fujisawa, K. Itoh, H. Iguchi, K. Matsuoka, S. Okamura, A. Shimizu, T. Minami, Y. Yoshimura, K. Nagaoka, C. Takahashi, M. Kojima, H. Nakano, S. Ohsima, S. Nishimura, M. Isobe, C. Suzuki, T. Akiyama, K. Ida, K. Toi, S.-I. Itoh, and P. H. Diamond, Phys. Rev. Lett. 93, 165002 (2004).

${ }^{53}$ A. Fujisawa, K. Itoh, H. Iguchi, K. Matsuoka, S. Okamura, A. Shimizu, T. Minami, Y. Yoshimura, K. Nagaoka, C. Takahashi, M. Kojima, H. Nakano, S. Ohshima, S. Nishimura, M. Isobe, C. Suzuki, T. Akiyama, K. Ida, K. Toi, S.-I. Itoh, P. H. Diamond, CHS group, Y. Hamada, A. Nishizawa, T. Ido, T. Watari, Y. Kawasumi, and the JIPPT-IIU Group, 20th IAEA Conference on Fusion Energy (IAEA, Portugal, 2004), Paper EX8-5Rb.

${ }^{54}$ G. R. McKee, R. J. Fonck, M. Jakubowski, K. H. Burrell, K. Hallatschek, R. A. Moyer, D. L. Rudakov, W. Nevins, G. D. Porter, P. Schoch, and X. $\mathrm{Xu}$, Phys. Plasmas 10, 1712 (2003).

${ }^{55}$ C. Schröder, O. Grulke, T. Klinger, and V. Naulin, Phys. Plasmas 11, (2004) 4249.

${ }^{56}$ C. Holland, J. H. Yu, A. James, D. Nishijima, M. Shimada, N. Taheri, and G. R. Tynan, "Observation of turbulent-driven shear flow in a cylindrical laboratory plasma device," Phys. Rev. Lett. (submitted).

${ }^{57}$ U. Stroth, "Turbulence studies in toroidal plasmas," presented at International Workshop on Structure Formation and Selection Rule in Turbulent Plasmas, RIAM Kyushu University, 2005. See also C. Lechte, "Microscopic structure of turbulence in TJ-K," Ph.D. thesis, University of Kiel (2003).

${ }^{58}$ G. D. Conway, B. Scott, J. Schirmer, M. Reich, A. Kendl, and the ASDEX Upgrade Team, Plasma Phys. Controlled Fusion 47, 1165 (2005).

${ }^{59}$ M. Hirsch, J. Baldzuhn, H. Ehmler, E. Holzhauer, and F. Wagner, Plasma Phys. Controlled Fusion 48, S155 (2006).

${ }^{60}$ K. H. Burrell, Plasma Phys. Controlled Fusion 48, A347 (2006). See also, V. Sokolov, X. Wei, A. K. Sen, and K. Avinash, ibid. 48, S111 (2006), in which novel techniques and experiments to confirm the structure of zonal flows are developed.

${ }^{61}$ G. R. McKee, D. K. Gupta, R. J. Fonck, D. J. Schlossberg, M. W. Shafer, and P. Gohil, Plasma Phys. Controlled Fusion 48, S123 (2006).

${ }^{62}$ T. Ido, Y. Miura, K. Hoshino, K. Kamiya, Y. Hamada, A. Nishizawa, Y. Kawasumi, H. Ogawa, Y. Nagashima, K. Shinohara, Y. Kusama, and JFT-2M group, Nucl. Fusion 46, 512 (2006).

${ }^{63}$ V. A. Vershkov, D. A. Shelukhin, S. V. Soldatov, A. O. Urazbaev, S. A. Grashin, L. G. Eliseev, A. V. Melnikov, and the T-10 team, Nucl. Fusion 45, S203 (2005).

${ }^{64}$ T. Ido, Y. Miura, K. Kamiya, Y. Hamada, K. Hoshino, A. Fujisawa, K. S.-I. Itoh, A. Nishizawa, H. Ogawa, Y. Kusama, and the JFT-2M group, Plasma Phys. Controlled Fusion 48, S41 (2006).

${ }^{65}$ G. R. Tynan, C. Holland, J. H. Yu, A. James, D. Nishijima, M. Shimada, and N. Taheri, Plasma Phys. Controlled Fusion 48, S51 (2006).

${ }^{66}$ E. Sánchez, C. Hidalgo, B. Gonçalves, C. Silva, M. A. Pedrosa, M. Hron, K. Erents, and JET EFDA contributors, J. Nucl. Mater. 337, 296 (2005).

${ }^{67}$ M. Kono and M. Tanaka, Phys. Rev. Lett. 84, 4369 (2000).

${ }^{68}$ G. S. Xu, B. N. Wan, J. Li, 20th IAEA Conference on Fusion Energy (IAEA, Portugal, 2004), Paper EX/8-4Rb.

${ }^{69}$ N. Vianello, V. Antoni, E. Spada, M. Spolaore, G. Serianni, R. Cavazzana, H. Bergsåker, M. Cecconello, and J. R. Drake, Nucl. Fusion 45, 761 (2005).

${ }^{70}$ Y. Kim and E. Powers, IEEE Trans. Plasma Sci. PS-7, 120 (1979).

${ }^{71}$ P. H. Diamond, M. N. Rosenbluth, E. Sanchez, C. Hidalgo, B. Van Milligen, T. Estrada, B. Brañas, M. Hirsch, and H. J. Hartfuss, Phys. Rev. Lett. 84, 4842 (2000).

${ }^{72}$ K. Itoh, Y. Nagashima, S-I Itoh, P. H. Diamond, A. Fujisawa, M. Yagi, and A. Fukuyama, Phys. Plasmas 12, 102301 (2005).

${ }^{73}$ Y. Nagashima, A. Ejiri, Y. Takase, K. Hoshino, K. Shinohara, K. Tsuzuki, K. Uehara, H. Kawashima, H. Ogawa, Y. Kusama, Y. Miura, and T. Ido, Phys. Rev. Lett. 95, 095002 (2005).

${ }^{74}$ Y. Nagashima, K. S.-I. Itoh Itoh, A. Fujisawa, K. Hoshino, Y. Takase, M. Yagi, A. Ejiri, K. Ida, K. Shinohara, K. Uehara, Y. Kusama, and the JFT-2M group, "Bispectral analysis applied to coherent floating potential fluctuations with geodesic acoustic wave frequency obtained in the edge plasmas on JFT-2M," Plasma Phys. Control. Fusion (submitted).

${ }^{75}$ Ch. P. Ritz, E. J. Powers, and R. D. Bengtson, Phys. Fluids B 1, 153 (1989).

${ }^{76}$ H. Xia and M. G. Shats, Phys. Plasmas 11, 561 (2004).

${ }^{77}$ Y. Nagashima, Y. Nagashima, K. Itoh, S.-I. Itoh, K. Hoshino, A. Fujisawa, A. Ejiri, Y. Takase, M. Yagi, K. Shinohara, K. Uehara, Y. Kusama, and JFT-2M group, "Observation of coherent bicoherence and biphase in potential fluctuations around geodesic acoustic mode frequency on JFT-2M," presented at 10th IAEA Technical Meeting on H-mode Physics and Transport Barriers (Ioffe Physico-Technical Institute, St. Petersburg, Russia, 2005).

${ }^{78}$ K. Itoh, K. Hallatschek, and S.-I. Itoh, Plasma Phys. Controlled Fusion 47, 451 (2005).

${ }^{79}$ A. Fujisawa, A. Shimizu, H. Nakano, S. Ohsima, K. Itoh, H. Iguchi, Y. Yoshimura, T. Minami, K. Nagaoka, C. Takahashi, M. Kojima, S. Nishimura, M. Isobe, C. Suzuki, T. Akiyama, Y. Nagashima, K. Ida, K. Toi, S.-I. K. Itoh Matsuoka, and S. Okamura, "Turbulence and transport characteristics on a transport barrier in a toroidal plasma," Plasma Phys. Controlled Fusion (in press).

${ }^{80}$ J. A. Krommes and C.-B. Kim, Phys. Rev. E 62, 8508 (2000).

${ }^{81}$ S.-I. Itoh and K. Itoh, J. Phys. Soc. Jpn. 69, 427 (2000).

${ }^{82}$ S.-I. Itoh, "Structure Formation and Dynamics in Turbulence," presented at the 60th Annual Meeting of the Physics Society of Japan, Tokyo University of Science, 2005, 25pXB-1.

${ }^{83}$ P. H. Diamond, "Physics of Zonal Flows," presented at the 60th Annual Meeting of the Physics Society of Japan, Tokyo University of Science, $2005,25 \mathrm{pXB}-2$.

${ }^{84}$ K. Mima, "Turbulence and structure in dense plasmas," presented at 60th Annual Meeting of Physics Society of Japan, Tokyo University of Science, 2005, 25pXB-3. 